\title{
Dental Phobia, Summary of Information Published on this Term
}

\author{
Ilma Robo ${ }^{1 *}$, Edison Shimaj', Arjon Hysaj ${ }^{2}$, Saimri Heta ${ }^{3}$ and Edlira Sadiku ${ }^{2}$ \\ ${ }^{1}$ Department of Periodontology, Faculty of Medical Sciences, Albanian University, Tirana, Albania \\ ${ }^{2}$ Private Dental Clinic, Tirana, Albania \\ ${ }^{3}$ QSUT, Pediatric Surgery, Pediatric Surgeon, Tirana, Albania
}

*Corresponding author: Ilma Robo, Department of Periodontology, Faculty of Medical Sciences, Albanian University, Tirana, Albania

\begin{tabular}{|c|c|}
\hline ARTICLE INFO & ABSTRACT \\
\hline Received: 玤 September 09, 2020 & Introduction: The article aims to provide an overview of information related to \\
\hline Published: 慧 September 24, 2020 & $\begin{array}{l}\text { dental phobia. The article is structured in the form of a mini review where paragraphs } \\
\text { are presented regarding various clinical aspects of dental phobia. There is a lot of talk }\end{array}$ \\
\hline $\begin{array}{l}\text { Citation: Ilma Robo, Edison Shimaj, Arjon } \\
\text { Hysaj, Saimri Heta, Edlira Sadiku. Dental }\end{array}$ & $\begin{array}{l}\text { treatment of patients who already appear in dental clinics with the symptoms of dental } \\
\text { phobia. }\end{array}$ \\
\hline hobia, Summary of Information Published & Conclusions: knowing full well that the patient suffers from dental phobia can prevent \\
\hline $\begin{array}{l}\text { on this Term. Biomed J Sci \& Tech Res } \\
30(4)-2020 . \text { BJSTR. MS.ID.004994. }\end{array}$ & $\begin{array}{l}\text { any unexpected thing that may occur as a result of the deterioration and appearance of } \\
\text { organic symptoms of dental phobia. In order to reach this point of patient confidence in }\end{array}$ \\
\hline $\begin{array}{l}\text { Keywords: Dental Phobia; "Bad" } \\
\text { Experiences; Anxiety Dental Patients; } \\
\text { Treatment of Anxiety Patients }\end{array}$ & $\begin{array}{l}\text { providing information about existing dental phobia, the initial communication and the } \\
\text { creation of bridges of communication with the patient, being the first meeting, should be } \\
\text { as healthy as possible. }\end{array}$ \\
\hline
\end{tabular}

Treatment of Anxiety Patients

\section{Introduction}

Dental phobia in most published articles is defined as exaggerated Unconscious fear, mainly by the dentist, but also by dental procedures or dental instruments. The objections to this definition are based on the fact that this fear is "unconscious", because dental phobia is mainly caused by previous "bad" experiences or badly lived and experienced in the patient, from a health point of view.Furthermore, there are some types of dental phobias that have not been properly described and categorized to date, for example "fear of the dentist" versus "fear of certain specific dental procedures", needle phobia and dental phobia from previous abuses which probably have nothing to do with this. Not all these fears can be considered unconscious[1,2].

Diagnostic criteria for specific phobias include:

1. A constant fear of a specific object or situation which is an extreme fear, or an unconscious fear,
2. An immediate anxiety at the moment of exposure to the stimulus of fear, which may take the form of panic,

3. Recognizing that fear is extreme and unconscious,

4. Avoiding the situation that produces anxiety,

5. Phobia affects normal functioning and causes a lot of stress.

\section{Characteristics and Causes of Dentophobia}

1. Characteristics of dental phobia

2. Disturbed sleep at night before dental examination

3. Increased nervousness while staying in the waiting room of the dental clinic

4. Crying or feeling like you are physically ill when you think about meeting the dentist 
5. Very disturbed when thinking or more precisely or at moment when dental objects are placed in the mouth during the dental visit, the patient feels as if it is difficult to breathe.

\section{Causes of Dental Phobia}

Dental phobia is usually caused by traumatic dental experiences, although there may be other causes as well, such as children often being influenced by their parents' fears and behaviors towards dental treatments. Some of the reasons for the lack of comfort that patients list as triggers of the subsequent situation of the appearance of dentophobia are:

1. Fear of needles (anesthesia)

2. Noise of dental milling cutters when working to open dental cavities

3. Aroma of the materials and solutions used, which prevails in dental clinics.

The most worrying problem for the appearance of dentophobia are those patients who:

1. Some time ago experienced an unpleasant experience,

2. Can't forget that bad experience they experienced

This bitter experience forces patients to stop going to the dentist, and even to abandon their oral health care altogether. For this reason, the dentist must play a very important role in stopping this fear of dental interventions, treating each patient individually, having patience and creativity during treatment, often becoming a good psychotherapist. And so, the patient by increasing confidence in his dentist, on the other hand, the fear decreases and over time, disappears completely.

It is worth mentioning that the patient should be talked to as much as possible with the patient and thus through informal conversations, makes the patient think of something else, thus minimizing the fear and even disappearing altogether.

Dental phobia may involve:

1. fear of dentists

2. fear of dental procedures

3. fear of pain

4. fear of injections or fear that the injection will not work properly

5. fear of the side effects of anesthesia.

The feeling of a situation that has no solution and the loss of control, as well as objects and other situations which remind the person of the phobic situation. The dentist is usually a major cause of these fears.

\section{Treatment of Anxiety Patients}

The World Health Organization (WHO) describes the fear of the dentist as a real disease. The symptomatic picture is manifested by tachycardia accompanied by tremors and drop in blood pressure, sweating, nausea, suffocation and generally a strong anxiety. However, only a part of the population is subject to this pathology. What generally affects the tools are the form of anxious noise, feminine traumatic experience or fear of dental intervention. These can push the patient to reduce dental treatment. Analgesic or antibiotic therapy is often used, which delays the solution of the problem, with the present danger and with a deterioration not only of chewing and the digestive apparatus, but also of the facial aesthetics[3,4].

Dentophobia can have consequences for dental health and wellbeing in general.

1. Pain in the teeth, gums and alveolar bones can be complicated. This is actually felt by aesthetics. Many frightened patients are ashamed of the issue of decayed teeth and bad breath they have.

2. They often do not have the courage to talk about their horrors, because in the past they were hidden by their acquaintances or directly by their dentist. In this way a vicious circle is created that does nothing but only worsens the situation.

3. As stated above, dental phobia is associated with various traumatic childhood experiences of the person who suffers. This means that dental phobia is learned, so it is the result of coping with situations which have caused the person to suffer as a result of dental interventions. Since these cases may have been numerous or even frequent, the person is taught that every time he goes to the dentist, he feels pain and from this the dentist is associated with the pain.

4. Thus, a fear of contact or going to the dentist is developed with the pain that the person may feel. This is reinforced by the fact that every time the person goes to the dentist and faces a dental intervention, he feels pain.

5. Since dental phobia is created as a result of learning, it can be removed.

Thus, the person, facing more satisfactory and less painful situations from contact or dental intervention, he begins to get used to the other fact: that not always going to the dentist causes pain. Thus, by eliminating the first condition the person begins to condition in another way, so he begins to overcome his fear of the dentist.

a. Like any fear and dread of the dentist, it relies on illogical assumptions.Removing the fear of anxious patients 
b. from the noise of clinic equipment can be reached

c. from screens placed in front of the dentist armchair connected to the DVD. The patient's attention is distracted both visually and audibly. The music suppresses the noise of the devices, and thus the patient's fear goes away.

d. For very excited people, pillows that massage the back and legs can be used, or with the option of heating to relax the patient.

e. Fear of the dentist is initially driven by the setting of the visit.

The colors and objects used in the decoration are placed in order to relax the patient.The dentist should explain step by step the treatment to be followed and should ask the patient to tell him when he is in pain and to give him confidence that he will stop the work when he is in pain.For this purpose, a strategy used by an Italian clinic "Studio dentistico Cardarelli" is:

1. Psychological support to reduce stress

2. Non-invasive treatment (with injections) for pain relief

3. Conscious treatment.

In the first place, it is the meaning of knowing an anxious patient in advance and treating him constantly in order to finally overcome the fear. To gradually achieve this goal, it is necessary to install a relationship of trust between the patient and the dentist.

\section{Non-Invasive Therapy (without Injections) Against Pain}

A stress-free and soothing atmosphere before, during and after treatment is a significant benefit.

Other elements that are part of our concept are the elimination of useless time, different and interesting reading materials in the waiting room, pleasant music in well-lit and modern places, where the typical "dentist smell" is distinguished. For patients suffering from dentophobia can help the first meeting held not in an operating room but in a "neutral" office without dental equipment $[5,6]$.

It is a non-invasive treatment and crucial and neutral pain reliever. For example, for single tooth care, it can be done without traditional anesthesia at first, replacing it with modern intraligamentary anesthesia (Peripess).

Benefit for patients:

1. no needle drilling.

2. painless virtual application.

3. immediate effect.

4. no anesthetic effect on lips, cheeks or tongue.

\section{Conscious Quenching with Nitric Oxide- Rebirth of a Method}

The anesthetic and sedative effect of gas (N2O chemicals, nitrous oxide) has been discovered since the 800s. Then it has been used as a narcotic gas millions of times. If, before local anesthesia instructions in dentistry, the analgesic effect of nitric oxide was in the foreground, today it is its anxiety-reducing and calming effects that make it an excellent tool for treating phobic patients.In the US and many other countries (England, Australia, Scandinavia) more than $50 \%$ of dentists regularly use nitrous oxide.

\section{How does Conscious Sedation Work?}

A mixture of oxygen and nitrous oxide is prepared through a small nasal mask.

Immediately after a few breaths, the effect begins with a feeling of relief and calm, the patient reaches a state of calm. He always remains conscious and aware of his feelings, but still feels a need to close his eyes. Fears and tension are transformed into a feeling of comfort and security. The special headphone waits for unpleasant treatment noises and ends with. A suggestive and relaxing music that reinforces the hypnotic effect of gas. The patient can be completely relaxed, leaving only pleasant thoughts to be shared, while time passes quickly. This perfect way of treatment represents a crucial anesthetic advantage over other methods.

\section{Ideal for Children as Well}

Children are always a big problem for parents and the dentist. If many adults are already abandoning dental treatment out of fear, how can we reduce this abandonment in children?Nitric oxide is an ideal aid for treating frightened children, also allowing children to be able to breathe voluntarily through the nose (starting at approximately 6-7 years old) and for the nose to be free, not closed. Thanks to the strong and anxiety-reducing action of gas, $80 \%$ of children who refuse a dental treatment can be treated. The reduction of disgusting reflexes, maintain full consciousness, also allows the full realization of dental intervention that the abovementioned children often do not accept. In many cases, thanks to conscious sedation, a treatment with total anesthesia can be avoided[7].

\section{Risks and Side Effects}

Conscious relaxation is a completely safe method. In the millions of documented cases in the USA, where it is used by about $50 \%$ of dentists, there have been no inappropriate cases. However, the method is not suitable for patients who have difficulty breathing[8].

1. The benefit of using conscious sedation

2. Psychological support to reduce stress 
3. Non-invasive treatment (without injections) pain relief

4. Conscious treatment

A person with such a phobia has erred in part of the process or in social learning, as a result of erroneous learning has reinforced the wrong processes. Every repeated action has continued to intensify, as a result of which wrong behaviors have been formed and this is the cause of the clinical picture. These wrong behaviors, which have been reinforced, need to be identified and worked on to correct them. Behaviors are not just some simple processes learned, but can also be chain links, involving conditioned stimuli from the environment or objects.

\section{Conclusions}

Conversation with the patient respecting the basic rules of bodily speech, especially in taking the anamnesis, are elements that should not be neglected during the first meeting with the patient. This communication significantly reduces the fear of the dentist but also of the environment where the patient is self-provided. The second element is the correct explanation of the procedures and the performance and achievement of adequate anesthesia to the patient, thus avoiding the immediate pain, followed by the sudden outpouring of significant amounts of internal adrenaline that leads to the consequences and the physical-organic reactions of the human body.The latest or most recently manufactured apparatus for achieving adequate anesthesia allows treatment that calms without the consequences of fear or dental phobia in patients.

\section{References}

1. Bowman U, Carlsson V, Westin M, Hakeberg M (2013) Psychological treatment of dental anxiety among adults: a systematic review. Wide. Eur J Oral Sci 121(3 Pt 2): 225-34.

2. Bray A, Chhun A, Donkersgoed R, Hoover S, Levitan S (2009) An evidence-based report investigating the most effective method to reduce dental anxiety. Clin Cosmet Investig Dent 8: 35-50.

3. Dumitrache MA, Neacsu V, Sfeatcu IR (2014) Efficiency of cognitive technique in reducing dental anxiety. Procedia Soc Behav Sci 149: 302-6.

4. Heaton LJ (2013) Behavioral interventions may reduce dental anxiety and increase acceptance of dental treatment in dentally fearful adults. J Evid Based Dent Pract 13(4): 160-162.

5. Getka EJ, Glass CR (1992) Behavioral and cognitive-behavioral approaches to the reduction of dental anxiety. Behav Ther 23(3): 43348.

6. Deva Priya Appukuttan (2016) Strategies to manage patients with dental anxiety and dental phobia: literature review. Clin Cosmet Investig Dent 8: 35-50.

7. Ian Paul Cardarelli (2016) Studiodentistico Cardarelli, Via Luca Belludi, Padova.

8. Sinha E, Rekha R, Nagashree SR (2019) Anxiety of dental treatment among patients visiting primary health centers. J Indian Assoc Public Health Dent 17(3): 235-45.
ISSN: 2574-1241

DOI: 10.26717/BJSTR.2020.30.004994

Ilma Robo. Biomed J Sci \& Tech Res

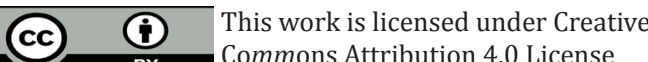

Submission Link: https://biomedres.us/submit-manuscript.php

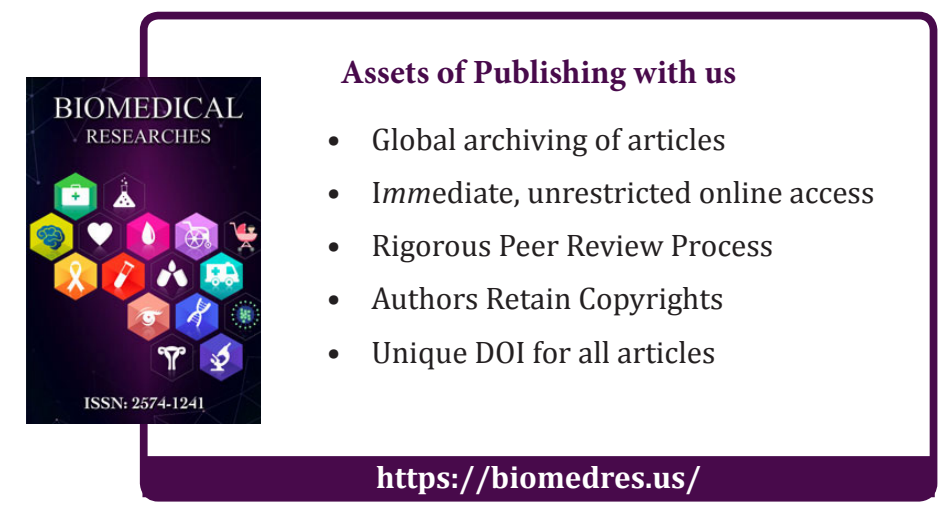

